\title{
Healthy food availability in small urban food stores: a comparison of four US cities
}

\author{
Melissa Nelson Laska ${ }^{1, *}$, Kelley E Borradaile ${ }^{2}$, June Tester ${ }^{3}$, Gary D Foster ${ }^{2}$ and \\ Joel Gittelsohn ${ }^{4}$ \\ ${ }^{1}$ Epidemiology \& Community Health, University of Minnesota, 1300 S. 2nd Street, WBOB Suite 300, \\ Minneapolis, MN 55454-1015, USA: ${ }^{2}$ Center for Obesity Research and Education, Temple University, \\ Philadelphia, PA, USA: ${ }^{3}$ Children's Hospital \& Research Center Oakland, Oakland, CA, USA: ${ }^{4}$ Center for \\ Human Nutrition, Johns Hopkins University, Baltimore, MD, USA
}

Submitted 5 March 2009: Accepted 26 0ctober 2009: First published online 8 December 2009

\begin{abstract}
Objective: Given that small food stores may be important retail food sources in low-income urban communities, our objective was to examine cross-city comparative data documenting healthy food availability within such facilities, particularly those located in low-income areas and nearby schools.

Design: Food stores in Baltimore, Maryland; Minneapolis/St. Paul, Minnesota; Oakland, California; and Philadelphia, Pennsylvania were selected for assessment based on proximity to low-income schools. Stores were defined as: (i) single-aisle ( $n$ 45); (ii) small ( $2-5$ aisles; $n 52$ ); and (iii) large ( $\geq 6$ aisles; $n$ 8). Staff conducted in-store audits to assess the presence/absence of twenty-eight healthy items, organized within five categories: (i) fresh fruits/vegetables, (ii) processed fruits/ vegetables, (iii) healthy beverages/low-fat dairy, (iv) healthy snacks and (v) other healthy staple foods.

Results: The availability of healthy food items was low, particularly in single-aisle and small stores, and there was significant cross-site variability in the availability of healthy snacks $(P<0 \cdot 0001)$ and other healthy staple foods $(P<0 \cdot 0001)$. No cross-site differences existed for fruits/vegetables or healthy beverages/low-fat dairy availability. Healthy food availability scores increased significantly with store size for nearly all food/beverage categories $(P<0 \cdot 01)$.

Conclusions: Overall, healthy food availability in these venues was limited. Region-specific factors may be important to consider in understanding factors influencing healthy food availability in small urban markets. Data suggest that efforts to promote healthy diets in low-income communities may be compromised by a lack of available healthy foods. Interventions targeting small stores need to be developed and tailored for use in urban areas across the USA.
\end{abstract}

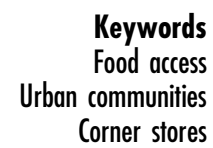

Keywords

communities

Corner stores
A growing body of research has documented widespread disparities in food availability across the USA ${ }^{(1)}$. For example, numerous studies have shown that the number and type of food stores available in neighbourhoods varies based on area-level socio-economic status (SES) and racial composition, such that supermarkets more likely locate in high-SES and low-minority areas and convenience stores more likely locate in low-SES and high-minority areas (see reference (1) for a review of this literature). These disparities in food access may impact dietary intake and contribute to SES and racial disparities in population-wide chronic disease risk ${ }^{(1)}$.

To date, however, few studies have directly measured food availability in stores, relying instead on store type (e.g. supermarket, convenience store) as a proxy for the types of food available. Some evidence indicates that while larger stores, such as supermarkets, do tend to have higher availability of healthy foods, neighbourhood disparities are not solely limited to the presence or absence of supermarkets. Recent work in Baltimore showed that supermarkets in lower-SES, minority neighbourhoods offered fewer healthy foods than supermarkets in higherSES, Caucasian neighbourhoods ${ }^{(2)}$. Initiatives to improve healthy food availability in low-SES areas are clearly needed.

An increasing interest in small urban stores or "corner store' markets has highlighted the role of these retail facilities on food access within low-SES communities and their potential as intervention targets ${ }^{(3)}$. Despite this interest, little data exist on the various types of foods available within these facilities. Previous research suggests that families utilize urban corner stores for 
day-to-day food purchases and items for home meal preparation $^{(4)}$. Children also report using corner stores to purchase convenience foods and snacks before, during and after school ${ }^{(5)}$. With recent research suggesting that food retailers cluster around schools particularly in low-income areas $^{(6)}$, as well as national data indicating that minority children walk to school at higher rates than non-minority children $^{(7)}$, it is likely that children in low-income, minority communities may be particularly influenced by food environments surrounding schools, highlighting the importance of understanding healthy food and beverage availability especially among corner store markets located within close proximity to schools in these areas.

The objectives of the present research were: (i) to assess availability of selected healthy food and beverage items (including both convenience and snack items that could be promoted among children, as well as household items for family home food preparation) in urban food stores; (ii) to compare the availability of these products in four large US urban areas; and (iii) to compare the availability of these products in stores of various sizes. Stores were selected for inclusion in this work based on their proximity to low-income, urban schools.

\section{Methods}

\section{Sampling strategy}

As part of the Robert Wood Johnson Foundation Healthy Eating Research Corner Stores Working Group, audits of neighbourhood food stores were conducted in Baltimore, Maryland; Minneapolis/St. Paul, Minnesota; Oakland, California; and Philadelphia, Pennsylvania. Stores were selected for assessment based on their proximity to lowincome schools (characterized as those with $\geq 50 \%$ of students eligible to receive free or reduced-priced lunch). School proximity was used as a selection criterion in part due to the widespread interest in childhood obesity risk and the clustering of unhealthy food sources around schools ${ }^{(8)}$. In addition these audits were a post hoc supplement to several funded and/or ongoing research studies; thus site selection criteria were largely influenced by existing study protocols and varied between cities. Food stores within a quarter of a mile of thirteen low-income elementary schools in Oakland and six elementary schools in Baltimore were sampled. In Minneapolis and St. Paul, stores within half a mile of thirty-six secondary schools were assessed. In Philadelphia, stores were assessed within four-block proximity of ten K-8 schools.

\section{Store definitions}

Food stores were defined as: (i) single-aisle; (ii) small (2-5 aisles); and (iii) large ( $\geq 6$ aisles). Other stores selling food and beverages (e.g. gas stations, drug stores) were excluded from these analyses. In total 108 stores were assessed: forty-five in Baltimore (thirty-four single-aisle, ten small, one large), sixteen in Minneapolis/St. Paul (none single-aisle, thirteen small, three large), twentyeight in Oakland (five single-aisle, nineteen small, four large) and nineteen in Philadelphia (six single-aisle, ten small, none large).

\section{Healtby food availability}

To achieve the study goals of estimating the presence of healthy items in urban food stores, the research team identified a list of twenty-eight food and beverage items to assess across the four sites. To maximize resource efficiency, the team developed a brief, easily administered assessment tool based on an existing tool from previous research ${ }^{(4)}$. Foods and beverages were selected that offered more nutrient-dense and/or less energy-dense alternatives to products typically available in small, urban markets. The goal was to identify relatively healthful foods that could be located (and possibly promoted) in urban markets, including both those that are readily consumed (e.g. ready-to-eat) and those that could be purchased for later preparation and/or consumption at home. Staff at each site conducted in-store audits to assess the presence or absence of these twenty-eight items. Items were organized within five larger categories: (i) fresh fruits/vegetables; (ii) processed fruits/ vegetables; (iii) healthy beverages/low-fat dairy; (iv) healthy snacks; and (v) other healthy staple foods. Table 1 defines each of these groups and the specific items included.

\section{Statistical analyses}

For each food/beverage category, a score was created consisting of the sum of items that were present. The presence/absence of items within each of the five mutually exclusive categories was averaged across stores, resulting in a mean number of healthy items available within each category. Higher numbers indicated a greater presence of healthy items. Analyses were replicated by city and then by store size, after collapsing across city. Secondary analyses examined the presence/absence of specific healthy food and beverage items. Since data collected at the store level represented the count of healthy items, Poisson regressions were used to compare the healthy items by site and store size. A conservative $\alpha$ level of $0 \cdot 01$ was considered statistically significant to account for the multiple comparisons examined in the work. All statistical analyses were conducted using the SAS statistical software package version $9 \cdot 2$ (SAS Institute Inc., Cary, NC, USA).

\section{Results}

Healthy food items were found in food stores at each site. Overall, the availability of fresh fruits and vegetables was low, with only $50 \cdot 0 \%$ of stores having $\geq 1$ fresh fruit/ vegetable, particularly compared to processed (e.g. frozen, canned) fruits and vegetables, which were available in $84 \cdot 3 \%$ of stores. The most commonly observed item 
Table 1 Healthy foods checklist applied during the urban food availability assessment across four US cities, 2008

\begin{tabular}{|c|c|}
\hline Category & Food/beverage items included \\
\hline Fresh fruit \& vegetables (including ready-to-eat products) & $\begin{array}{l}\text { Fresh fruit } \\
\text { Fresh vegetables } \\
\text { Pre-packaged salads }\end{array}$ \\
\hline Processed fruit \& vegetables & $\begin{array}{l}\text { Frozen (or canned) vegetables } \\
\text { Canned fruit (in light syrup or juice only) } \\
\text { No-added-sugar apple sauce }\end{array}$ \\
\hline Healthy beverages \& low-fat dairy & $\begin{array}{l}\text { Bottled/flavoured water } \\
100 \% \text { fruit juice } \\
1 \% \text { or skimmed milk } \\
\text { Low- or reduced-fat cheese ( }<10 \% \text { DV for fat) } \\
\text { Low-fat or non-fat yoghurt ( }<10 \% \text { DV for fat) }\end{array}$ \\
\hline Healthy snacks & $\begin{array}{l}\text { Low-fat crackers ( }<10 \% \text { DV for fat) } \\
\text { Nuts } \\
\text { Low-fat popcorn ( }<10 \% \text { DV for fat) } \\
\text { Low-fat trail mix and/or dried fruit }(<10 \% \text { DV for fat) } \\
\text { Low-sugar/low-fat granola bars ( } 10 \mathrm{~g} \text { sugar, } \geq 10 \% \text { DV for fibre) } \\
\text { Baked or low-fat potato chips } \\
\text { Pretzels } \\
\text { Graham crackers or Animal crackers }\end{array}$ \\
\hline Other healthy staple foods & $\begin{array}{l}\text { Peanut butter } \\
\text { High-fibre bread ( } \geq 10 \% \text { DV for fibre) } \\
\text { Brown rice } \\
\text { Beans or chickpeas } \\
\text { Lentils } \\
\text { High-fibre cereal ( } \geq 10 \% \text { DV for fibre) } \\
\text { Low-sugar cereal ( }<10 \mathrm{~g} \text { sugar) } \\
\text { Low-sugar pudding packs } \\
\text { Jello } \\
\text { Pre-made sandwiches }\end{array}$ \\
\hline
\end{tabular}

DV, daily value.

available in the 'healthy beverages and low-fat dairy' category was bottled/flavoured water, available in $94.4 \%$ of stores. For healthy snacks, nuts and pretzels were the most frequently observed items (in $78.7 \%$ and $60 \cdot 2 \%$ of stores, respectively), and beans/lentils/chickpeas and peanut butter were the most frequently observed staple foods (in $69 \cdot 4 \%$ and $52 \cdot 8 \%$ of stores).

Findings suggest notable variability in healthy food availability across the four sites (Table 2). For example, in examining stores of all sizes, there was significant crosssite variability in the availability of healthy snacks $(P<0 \cdot 0001)$ and other healthy staple foods $(P<0 \cdot 0001)$. Similar trends were also apparent when analyses were limited to the cross-site examination of small stores only (i.e. those with 2-5 aisles). In general, stores in Minneapolis/St. Paul tended to have the highest availability of these healthful products, whereas Baltimore stores had the lowest availability. No significant differences existed between the sites for fresh fruits/vegetables, processed fruits/vegetables and healthy beverages/low-fat dairy.

However, in examining the availability of individual food/beverage items, the availability varied widely. For example, skimmed or $1 \%$ milk was carried in only $6.7 \%$ of stores in Baltimore $v .81 \cdot 3 \%$ in Minneapolis/St. Paul. Similarly, baked potato chips were found in none of the stores in Baltimore, compared with 10.5\% in Philadelphia, $37.5 \%$ in Minneapolis/St. Paul and $92.9 \%$ in Oakland. Additional analyses comparing the availability of items in small stores only across all sites (to account for the variation in store size by site) yielded similar findings. Overall, however, some food items were more consistently available across sites (such as pretzels, available in $42.9 \%$ of stores in Oakland, $51 \cdot 1 \%$ in Baltimore, $81 \cdot 3 \%$ in Minneapolis/St. Paul and $89.5 \%$ in Philadelphia).

Collapsing all of the data across the four sites, findings also indicate that healthy food availability varied by store size. Availability scores increased significantly with store size for most food/beverage categories, including fresh fruits/vegetables $(P=0 \cdot 002)$, healthy beverages/low-fat dairy $(P=0 \cdot 007)$, healthy snacks $(P<0 \cdot 0001)$ and other healthy staple foods $(P<0 \cdot 0001)$. Only $11 \cdot 1 \%$ of singleaisle stores had skimmed or $1 \%$ milk, compared with $38.2 \%$ of small and $100.0 \%$ of large stores. Similarly, baked potato chips were found in $8.9 \%$ of single-aisle, $43.6 \%$ of small and $75.0 \%$ of large stores.

\section{Discussion}

These findings indicate that although healthy food/beverage items were found in at least some stores at each of the four sites, overall healthy food availability was limited. For example, fresh fruits/vegetables were available in only $50 \cdot 0 \%$ of all stores, and skimmed or low-fat milk was available in $32.0 \%$ of stores. These data suggest that the efforts of low-SES groups to eat well and improve dietary 
Table 2 Healthy food and beverage availability in urban stores located in close proximity to schools of low socio-economic status across four US cities, 2008

\begin{tabular}{|c|c|c|c|c|c|}
\hline & $\begin{array}{c}\text { Fresh fruit \& } \\
\text { vegetables }\end{array}$ & $\begin{array}{l}\text { Processed fruit \& } \\
\text { vegetables }\end{array}$ & $\begin{array}{l}\text { Healthy beverages } \\
\text { \& low-fat dairy }\end{array}$ & $\begin{array}{l}\text { Healthy } \\
\text { snacks }\end{array}$ & $\begin{array}{l}\text { Other healthy } \\
\text { staple foods }\end{array}$ \\
\hline Possible range & $0-3$ & $0-3$ & $0-5$ & $0-8$ & $0-9$ \\
\hline \multicolumn{6}{|l|}{ All stores, by site } \\
\hline Baltimore, MD ( $n$ 45) & $0 \cdot 7$ & $1 \cdot 3$ & $2 \cdot 2$ & $1 \cdot 6$ & $2 \cdot 3$ \\
\hline Minneapolis/St. Paul, MN ( $n$ 16) & $1 \cdot 1$ & $2 \cdot 1$ & $3 \cdot 3$ & 4.9 & $4 \cdot 8$ \\
\hline Oakland, CA ( $n$ 28) & $1 \cdot 1$ & $1 \cdot 3$ & $2 \cdot 4$ & 3.5 & $2 \cdot 8$ \\
\hline Philadelphia, PA ( $n$ 19) & $0 \cdot 8$ & $1 \cdot 6$ & $2 \cdot 1$ & $3 \cdot 8$ & $3 \cdot 9$ \\
\hline$P$ value (across sites) & $0 \cdot 19$ & $0 \cdot 16$ & 0.08 & $<0.0001$ & $<0.0001$ \\
\hline \multicolumn{6}{|l|}{ Small stores only, by site } \\
\hline Baltimore, MD (n 10) & $1 \cdot 1$ & $1 \cdot 5$ & $2 \cdot 3$ & $2 \cdot 0$ & $3 \cdot 4$ \\
\hline Minneapolis/St. Paul, MN ( $n$ 13) & $1 \cdot 3$ & $2 \cdot 1$ & $3 \cdot 1$ & $4 \cdot 6$ & $4 \cdot 5$ \\
\hline Oakland, CA $(n 19)$ & $1 \cdot 1$ & $1 \cdot 6$ & $2 \cdot 3$ & $3 \cdot 6$ & $2 \cdot 8$ \\
\hline Philadelphia, PA ( $n$ 13) & $0 \cdot 8$ & $1 \cdot 6$ & $2 \cdot 2$ & $3 \cdot 8$ & $4 \cdot 2$ \\
\hline$P$ value (across sites) & $0 \cdot 73$ & 0.69 & 0.45 & 0.007 & 0.06 \\
\hline \multicolumn{6}{|l|}{ All sites, by store size } \\
\hline Single-aisle ( $n$ 45) & 0.5 & $1 \cdot 1$ & $2 \cdot 0$ & $1 \cdot 8$ & $1 \cdot 9$ \\
\hline Small (2-5 aisles) ( $n$ 55) & $1 \cdot 1$ & $1 \cdot 7$ & $2 \cdot 5$ & $3 \cdot 6$ & $3 \cdot 7$ \\
\hline Large ( $\geq 6$ aisles) ( $n$ 8) & $1 \cdot 4$ & $1 \cdot 9$ & $4 \cdot 0$ & $5 \cdot 4$ & $5 \cdot 5$ \\
\hline$P$ value (across store types) & 0.002 & 0.04 & 0.007 & $<0.0001$ & $<0.0001$ \\
\hline
\end{tabular}

intake may be compromised by the lack of healthy food available in urban markets.

Healthy food availability (particularly healthy snacks and staple foods) was also highly variable across the sites. Although we had hypothesized that geographic variability may be due to the types of stores present in each city (e.g. a high number of single-aisle stores in Baltimore), these trends were observed even after limiting our analyses to small stores only. It is possible that geographic disparities in food access may be due to disparities in area-level SES across sites. For example, median household income (in 2008 inflation-adjusted US dollars) ranged from \$36222 in Philadelphia to \$39083 in Baltimore, \$47097 in Minneapolis to $\$ 48596$ in Oakland ${ }^{(9)}$. Differences in the size or capacity of food distribution chains used by owners of small stores across different geographic regions may also contribute to these disparities, and additional research is needed to better understand these distribution systems. Furthermore, racial and cultural issues across sites may be influential, particularly with storeowners of one ethnic group servicing a clientele predominantly from a different ethnic group. Such situations have led to storeowners fostering a wide range of misperceptions about the potential market for healthy food items ${ }^{(4)}$.

To our knowledge, the present study is among the first of its kind to provide a cross-city comparison of directly measured healthy food availability in urban stores located near low-SES schools. Despite its strengths, there are numerous limitations. These data represent a relatively small sample size of stores. More comprehensive investigations are needed to confirm these findings, particularly those that include non-food stores (e.g. drug stores, gas stations). Our data do not allow us to fully examine healthy food availability by sites $v$. store size, in that stores of various sizes were not equally dispersed across the sites. In addition, we did not assess other important issues, such as the quality/ freshness of available food, package size and item pricing, which may vary considerably across stores. Furthermore, given our goal to be parsimonious in our selection of foods/ beverages to assess in the study and to provide a quick and efficient means of data collection, these findings do not represent store availability of all possible food/beverage items. Other comprehensive nutrition environment assessment tools are available for use, but require substantial staff support for administration ${ }^{(10)}$. These tools have also been more rigorously tested than the checklist that was used in the present study. Finally, while the four cities included in our study represent a range of demographic characteristics, previous research has demonstrated that other populations may experience specific challenges not addressed here ${ }^{(11)}$. These need to be explored in future research.

Overall, these findings yield important insights for developing intervention strategies that target urban food stores serving low-income families and youths. Across all of the sites approximately half of all stores assessed here were currently stocking at least one type of fresh fruit or vegetable. It is important to help stores improve the quantity, quality and/or variety of fruits and vegetables that are for sale, and our findings suggest that many stores may have the capacity to stock these items. Thus, it may be possible to readily aid storeowners in increasing their availability of such products. Other items that showed a high degree of variability across sites, such as baked potato chips, do not require special handling or storage procedures, and thus could also be readily stocked by storeowners. Incorporating new products into regular inventory, including those requiring extensive temperature-controlled or refrigerated shelf space that may not be currently available (e.g. low-fat milk), may present additional barriers to storeowners that will need to be addressed.

In conclusion, small urban stores may serve as an important retail food source for low-SES families. A growing 
body of evidence has begun to suggest that health promotion efforts aimed at increasing healthy food availability in these settings are feasible ${ }^{(3)}$, but a wider range of interventions that target small, corner stores need to be developed and tailored for use in urban areas across the USA. Furthermore, it is essential that these strategies include systematic evaluation plans to document programme successes and failures and promote generalizable knowledge in the field.

\section{Acknowledgements}

Data collection in Baltimore, Philadelphia and Oakland was funded through grant support from the Robert Wood Johnson Foundation Healthy Eating Research Program. Additional salary support was provided by the National Cancer Institute (K07CA126837). There are no conflicts of interest to report. All authors contributed to data collection, interpretation of findings and revision of the manuscript. M.N.L. was responsible for drafting the manuscript and K.E.B. was responsible for analysing the data.

\section{References}

1. Larson NI, Story MT \& Nelson MC (2009) Neighborhood environments: disparities in access to healthy foods in the US. Am J Prev Med 36, 74-81.
2. Franco M, Diez Roux AV, Glass TA et al. (2008) Neighborhood characteristics and availability of healthy foods in Baltimore. Am J Prev Med 35, 561-567.

3. Song H, Gittelsohn J, Kim M et al. (2009) A corner store intervention in a low-income urban community is associated with increased availability and sales of some healthy foods. Public Health Nutr 12, 2060-2067.

4. Gittelsohn J, Franceschini M, Rasooly I et al. (2007) Understanding the food environment in a low-income urban setting: implications for food store interventions. $J$ Hunger Environ Nutr 2, 33-50.

5. Dodson J, Hsiao Y-C, Kasat-Shors M et al. (2009) Formative research for a healthy diet intervention among inner-city adolescents: the importance of family, school and neighborhood environment. Ecol Food Nutr 48, $39-58$.

6. Zenk SN \& Powell LM (2008) US secondary schools and food outlets. Health Place 14, 336-346.

7. McDonald NC (2007) Active transportation to school: trends among US schoolchildren, 1969-2001. Am J Prev Med 32, 509-516.

8. Austin SB, Melly SJ, Sanchez BN et al. (2005) Clustering of fast-food restaurants around schools: a novel application of spatial statistics to the study of food environments. Am J Public Health 95, 1575-1581.

9. US Census Bureau (2009) American FactFinder. http:// www.factfinder.census.gov/home/saff/main.html?_lang=en (accessed November 2009).

10. Glanz K, Sallis JF, Saelens BE et al. (2007) Nutrition Environment Measures Survey in stores (NEMS-S): development and evaluation. Am J Prev Med 32, 282-289.

11. Curran S, Gittelsohn J, Anliker J et al. (2005) Process evaluation of a store-based environmental obesity intervention on two American Indian Reservations. Health Educ Res 20, 719-729. 